\title{
A CHARACTERIZATION OF NORMAL EXTENSIONS FOR SUBFACTORS
}

\author{
TAMOTSU TERUYA
}

(Communicated by Palle E. T. Jorgensen)

Dedicated to Professor Tsuyosi Ando on his sixtieth birthday

\begin{abstract}
Let $N \subset M \subset L$ be a tower of factors. If $L$ is a crossed product $N \rtimes_{\alpha} G$ of $N$ by an outer action $\alpha$ of a finite group $G$ on $N$ then it is well known that there exists a subgroup $H$ of $G$ such that $M=N \rtimes_{\alpha_{\mid H}} H$. We prove in this paper that $H$ is a normal subgroup of $G$ if and only if there exist a finite group $F$ and an outer action $\beta$ of $F$ on $M$ such that $L=M \rtimes_{\beta} F$.
\end{abstract}

\section{INTRODUCTION}

Let $N \subset M \subset L$ be a tower of finite factors. In [NT] Nakamura and Takeda studied a Galois theory for finite factors. If $N$ is a fixed-point algebra $L^{G}$ of $L$ by an outer action $\alpha$ of a finite group $G$ on $L$ then there exists a subgroup $H$ of $G$ such that $M$ is a fixed-point algebra $L^{H}$. In [T] Takeda proved that $H$ is a normal subgroup of $G$ if and only if $N$ is a fixed point algebra $M^{F}$ of $M$ by an outer action $\beta$ of some finite group $F$ on $M$. We present in this paper a simple proof for arbitrary factors. To be more concrete, suppose that $L$ is a crossed product $N \rtimes_{\alpha} G$ of a factor $N$ by an outer action $\alpha$ of a finite group $G$ on $N$ and $M$ is a crossed product $N \rtimes_{\left.\alpha\right|_{H}} H$ of $N$ by the induced outer action $\left.\alpha\right|_{H}$ (by restriction of $\alpha$ ) of a subgroup $H$ of $G$ on $N$. We prove in this case that $H$ is a normal subgroup of $G$ if and only if there exist a finite group $F$ and an outer action $\beta$ of $F$ on $M$ such that $L$ is a crossed product $M \rtimes_{\beta} F$. Using Kosaki's characterization of crossed product [K] and examining the restriction-induction graph (for example, see $[\mathrm{KY}]$ or $[\mathrm{O}]$ ), one can also obtain the result. But we give a simple proof without examining higher relative commutants of $M \subset L$.

\section{THEOREM AND COROLLARIES}

Theorem. Let $N \subset M \subset L$ be a tower of factors. If $L$ is a crossed product $N \rtimes_{\alpha} G$ of $N$ by an outer action $\alpha$ of a finite group $G$ on $N$ then the subgroup $H$ of $G$ associated with $M$ is a normal subgroup of $G$ if and only if $L$ is a

Received by the editors June 16, 1992.

1991 Mathematics Subject Classification. Primary 46L37.

Key words and phrases. Factor, outer action, crossed product, Galois theory, fixed point algebra, conditional expectation. 
crossed product $M \rtimes_{\beta} F$ of $M$ by an outer action $\beta$ of some finite group $F$ on $M$. Furthermore, $F$ is the quotient group $G / H$.

Proof. Suppose that $H$ is a normal subgroup of $G$ and $\left\{u_{g}\right\}_{g \in G}$ are canonical unitaries of a crossed product. It is known that a 2-cocycle is a coboundary. (See [J1] or [S].) So we can choose the section $\theta: F=G / H \rightarrow G$ such that $\left\{u_{\theta(\sigma)}\right\}_{\sigma \in F}$ is a unitary representation of $F$. Observing that

$$
L=\left(M \cup\left\{u_{\theta(\sigma)}\right\}_{\sigma \in F}\right)^{\prime \prime}
$$

and $\beta_{\sigma}=A d u_{\sigma}$ is an outer action of $F$ on $M$, we have $L$ is a crossed product $M \rtimes_{\beta} F$ of $M$ by an outer action $\beta$ of $F$.

Conversely suppose that $L$ is a crossed product $M \rtimes_{\beta} F$ of $M$ by an outer action $\beta$ of some finite group $F$ on $M$. Then we have

$$
M=\left(N \cup\left\{u_{g} \mid g \in H\right\}\right)^{\prime \prime}, \quad L=\left(N \cup\left\{u_{g} \mid g \in G\right\}\right)^{\prime \prime},
$$

and

$$
L=\left(M \cup\left\{z_{\sigma} \mid \sigma \in F\right\}\right)^{\prime \prime}
$$

where $u_{g}$ and $z_{\sigma}$ are canonical unitaries of crossed products. We set $E_{M}$ : $L \rightarrow M$ as the unique conditional expectation such that

$$
E_{M}(X)=x_{e} \text { for } X=\sum_{\sigma \in F} x_{\sigma} z_{\sigma} \in L
$$

where $x_{\sigma}$ is a element of $M$ and $e$ is the unit element of $F$. Then we get

$$
u_{g}=\sum_{\sigma \in F} E_{M}\left(u_{g} z_{\sigma}^{*}\right) z_{\sigma}
$$

i.e., $\left\{z_{\sigma} \mid \sigma \in F\right\}$ are Pimsner-Popa type bases in $L$ with respect to $M$ (see [PP] or [W]). Therefore, there is $\sigma_{0} \in F$ with $E_{M}\left(u_{g} z_{\sigma_{0}}^{*}\right) \neq 0$. Since, for any $x \in N, x u_{g} z_{\sigma_{0}}^{*}=u_{g} z_{\sigma_{0}}^{*} z_{\sigma_{0}} u_{g}^{*} x u_{g} z_{\sigma_{0}}^{*}$ and $z_{\sigma_{0}} u_{g}^{*} x u_{g} z_{\sigma_{0}}^{*} \in M$, we have

$$
x E_{M}\left(u_{g} z_{\sigma_{0}}^{*}\right)=E_{M}\left(u_{g} z_{\sigma_{0}}^{*}\right) z_{\sigma_{c}} u_{g}^{*} x u_{g} z_{\sigma_{0}}^{*} \text { for } x \in N
$$

and

$$
E_{M}\left(u_{g} z_{\sigma_{0}}^{*}\right) z_{\sigma_{0}} u_{g}^{*} \in N^{\prime} \cap L=\mathbb{C}
$$

hence, $u_{g} z_{\sigma_{0}}^{*} \in M$. Therefore,

$$
u_{g} M u_{g}^{*}=u_{g} z_{\sigma_{0}}^{*} M z_{\sigma_{0}} u_{g}^{*}=M
$$

for any $g \in G$. This means, for any $g \in G$ and any $h \in H, u_{g} u_{h} u_{g}^{*}=u_{g h g^{-1}} \in$ $M$, so $g h g^{-1} \in H$. Thus $H$ is a normal subgroup of $G$, and by the first part of this proof we get $F=G / H$.

Using this theorem, we immediately obtain the next corollaries.

Corollary. Let $M$ be a factor and $K$ a fixed-point algebra $M^{G}$ by an outer action $\alpha$ of a finite group $G$ on $M$. If $H$ is a subgroup of $G$ and $N$ is a fixed-point algebra $M^{H}$ then $H$ is a normal subgroup of $G$ if and only if there exist a finite group $F$ such that $K$ is a fixed-point algebra $N^{F}$ of $N$ by an outer action $\beta$ of $F$ on $N$. Furthermore, $F$ is the quotient group $G / H$.

This corollary is an extension of Takeda's theorem in [T] to arbitrary factors. 
Proof. Suppose that $M$ acts on a Hilbert space $\mathscr{H}$ in a standard way. By the duality for an inclusion of factors, $K^{\prime}=M^{\prime} \rtimes G$ and $N^{\prime}=M^{\prime} \rtimes H$. (For example, see [GHJ].) By the previous theorem, $H$ is a normal subgroup of $G$ if and only if there exists a finite group $F$ such that $K^{\prime}=N^{\prime} \rtimes F$; i.e., $K$ is a fixed-point algebra $N^{F}$.

Corollary. With the same notation as the previous theorem, if $H$ is not a normal subgroup of $G$ then for any factor $K$ and any outer action $\beta$ of a finite group $F$ on $K$, neither $K \subset K \rtimes_{\beta} F$ nor $K^{F} \subset K$ is conjugate to $M \subset L$.

Proof. By the previous theorem, it is obvious that $M \subset L$ and $K \rtimes_{\beta} F$ are not conjugate. Suppose that $M \subset L$ and $K^{F} \subset K$ are conjugate. Let $N_{1}$ and $M_{1}$ be Jones's basic constructions [J2] of $L$ by $N$ and $M$ respectively. Since $N^{\prime} \cap N_{1}$ is isomorphic to $l^{\infty}(G)$ and $N^{\prime} \cap N_{1} \supset M^{\prime} \cap M_{1}, M^{\prime} \cap M_{1}$ is commutative. However, $M^{\prime} \cap M_{1}$ is isomorphic to a group von Neumann algebra $R(F)$. Therefore, $F$ is abelian. So we get

$$
K=K^{F} \rtimes_{\widetilde{\beta}} F
$$

where $\widetilde{\beta}$ is a dual action of $\beta$. It is a contradiction to the theorem.

\section{ACKNOWLEDGMENT}

I am greatly indebted to Professor Y. Watatani for his many useful suggestions and encouragement.

\section{REFERENCES}

[GHJ] F. Goodman, P. de la Harpe, and V. F. R. Jones, Coxeter graphs and towers of algebras, Math. Sci. Res. Inst. Publ., vol. 14, Springer-Verlag, New York, 1989.

[J1] V. F. R. Jones, Actions of finite groups on the hyperfinite type $\mathrm{II}_{1}$ factor, Mem. Amer. Math. Soc., no 237, Amer. Math. Soc., Providence, RI, 1980.

[J2] Index for subfactors, Invent. Math. 72 (1983).

[K] H. Kosaki, Characterization of crossed product (properly infinite case), Pacific J. Math. 137 (1989), 159-167.

[KY] H. Kosaki and S. Yamagami, Irreducible bimodules associated with crossed product algebras, Internat. J. Math. 3 (1992), 661-676.

[NT] N. Nakamura and Z. Takeda, A Galois theory for finite factors, Proc. Japan Acad. 36 (1960), 258-260.

[O] A. Ocneanu, Quantum symmetry, differential geometry of finite graphs and classification of subfactors, University of Tokyo seminary notes, 1991.

[PP] M. Pimsner and S. Popa, Entropy and index for subfactors, Ann. Sci. École Norm. Sup. (4) 19 (1986), 57-106.

[S] Colin E. Sutherland, Cohomology and extensions of von Neumann algebras. I, II, Publ. Res. Inst. Math. Sci. 16 (1980), 105-133, 135-174.

[T] Z. Takeda, On the normal basis theorem of the Galois theory for finite factors, Proc. Japan Acad. 37 (1961).

[W] Y. Watatani, Index for $C^{*}$-subalgebras, Mem. Amer. Math. Soc., no. 424, Amer. Math. Soc., Providence, RI, 1990.

Department of Mathematics, Faculty of Science, Hokkaido University, Sapporo 060, 